\title{
Pulse compression of submillijoule few-optical- cycle infrared laser pulses using chirped mirrors
}

\author{
Mathieu Giguère, ${ }^{1}$ Bruno E. Schmidt, ${ }^{1}$ Andrew D. Shiner, ${ }^{2}$ Marie-Andrée Houle, ${ }^{1}$ Heidi C. Bandulet, ${ }^{1}$ \\ Gabriel Tempea, ${ }^{3}$ David M. Villeneuve, ${ }^{2}$ Jean-Claude Kieffer, ${ }^{1}$ and François Légaré ${ }^{1, *}$ \\ ${ }^{1}$ Institut National de la Recherche Scientifique, Centre Énergie Matériaux et Télécommunications, \\ 1650 Boulevard Lionel-Boulet, Varennes, Quebec J3X1S2, Canada \\ ${ }^{2}$ National Research Council of Canada, 100 Sussex Drive, Ottawa ON K1A OR6, Canada \\ ${ }^{3}$ Femtolasers Produktions GmbH, Fernkorng. 10, A-1100 Vienna, Austria \\ *Corresponding author: legare@emt.inrs.ca
}

Received April 15, 2009; revised May 16, 2009; accepted May 19, 2009; posted May 22, 2009 (Doc. ID 110186); published June 12, 2009

We report generation of $400 \mu \mathrm{J}, 13.1 \mathrm{fs}, 1425 \mathrm{~nm}$ optical parametric amplifier laser pulses. Spectral broadening of a $100 \mathrm{~Hz}$ optical parametric amplifier laser source is achieved by self-phase modulation in an argonfilled hollow-core fiber, and dispersion compensation is performed using chirped mirrors. This laser source will be useful for ultrafast time-resolved molecular orbital tomography. (C) 2009 Optical Society of America OCIS codes: $320.5520,320.7090$.

Generation of intense few-optical-cycle laser pulses at $800 \mathrm{~nm}$ has been the key technological breakthrough for attosecond science-high-harmonic generation (HHG) [1-4] and pump-probe molecular imaging $[5,6]$. At the moment, using carrier-envelopephase (CEP)-stabilized 1.5 cycle Ti-Sa laser pulses and an interaction media with a large ionization potential (neon, Ip=21 eV), pulse duration of 80 attoseconds has been generated [7]. Since the cutoff of the harmonic spectra scales with $I \lambda^{2}$ ( $I$ is the laser intensity and $\lambda$ the laser wavelength) [8], fewoptical-cycle IR laser pulses are required to further decrease the duration of attosecond laser pulses [9]. Such development will also be highly beneficial for molecular orbital tomography, since shorter harmonic wavelength implies better spatial resolution [10].

For HHG, submillijoule of energy per laser pulse is required. At $800 \mathrm{~nm}$, submillijoule few-optical-cycle laser pulses are commonly generated using the combination of self-phase modulation (SPM) through propagation of submillijoule multicycle pulses in a hollow-core fiber filled with a noble gas followed by dispersion compensation using chirped mirrors $[11,12]$. This is the standard experimental approach for dispersion compensation of $800 \mathrm{~nm}$ few-opticalcycle laser pulses and used in many laboratories worldwide. In the IR, chirped mirrors have been used for dispersion compensation in Cr:forsterite oscillators [13] but never to compress broadband laser pulses resulting from nonlinear propagation in a hollow-core fiber setup. Recently, we demonstrated generation of stable broadband IR spectra using this approach, in which we used an acousto-optic programmable dispersive filter for dispersion compensation obtaining $20 \mathrm{fs}$ pulse duration at $1300 \mathrm{~nm}$ with $10 \mu \mathrm{J}$ of energy per pulse [14]. In this Letter, we demonstrate the generation and characterization of $400 \mu \mathrm{J}, 13.1 \mathrm{fs}, 1425 \mathrm{~nm}$ laser pulses by using IR chirped mirrors for dispersion compensation. This approach is a robust method to generate intense IR few-optical-cycle laser pulses and can be easily inte- grated with submillijoule femtosecond optical parametric amplifier (OPA) laser systems that are becoming widely available, and recently extended to sub-10 mJ level [15].

In the IR spectral range, few-optical-cycle laser pulses have been generated using other approaches that are generally more complex than the one proposed in this Letter: (1) $740 \mu \mathrm{J}, 15.6 \mathrm{fs}$ at $2.1 \mu \mathrm{m}$ using an optical parametric chirped-pulse amplifier [16]; (2) $270 \mu \mathrm{J}, 17 \mathrm{fs}$ at $2.1 \mu \mathrm{m}$ using pulse compression by filamentation [17]; and (3) $1.2 \mathrm{~mJ}, 17 \mathrm{fs}$ at $1.5 \mu \mathrm{m}$ using difference-frequency generation of CEP-stabilized few-optical-cycle $800 \mathrm{~nm}$ laser pulses followed by type II parametric amplification [18].

The experiment was conducted at the Advanced Laser Light Source using the $100 \mathrm{~Hz}, 100 \mathrm{~mJ}, 35 \mathrm{fs}$ Ti:Sa laser system (Thales Laser). Using $7 \mathrm{~mJ}$ of Ti:Sa, $1.2 \mathrm{~mJ}, 50 \mathrm{fs}, 1425 \mathrm{~nm}$ signal laser pulses are produced using an OPA based on a seed generated by parametric superfluorescence (HE-TOPAS, Light Conversion) with pulse to pulse stability of $2 \%-3 \%$ rms. At the output of the OPA, the pulse energy is attenuated using the combination of an achromatic half-wave plate with Glan-Calcite polarizer. The $1425 \mathrm{~nm}$ laser beam is coupled into the hollow-core fiber compressor using an $f=1 \mathrm{~m}$ plano-convex lens. The hollow-core fiber is supported on an aluminum V-groove and attached on both ends to closed gas cells. The hollow-core fiber is evacuated and filled with argon by maintaining $1.7 \mathrm{Atm}$ of pressure in both cells. At the output, the laser beam was collimated using a $1 \mathrm{~m}$ concave silver mirror. Typically, the optical transmission was near $40 \%$ owing to the poor spatial quality of the OPA laser beam.

Figure 1 shows typical spectra after the fiber for low (30 $\mu \mathrm{J}$, dashed-dotted curve) and high (400 $\mu \mathrm{J}$, solid curve) output energies (measured with NIR256 spectrometer from Ocean Optics, Inc.). At low energy, SPM is not observed, and the OPA signal spectra are unchanged upon propagation in the hollow-core fiber. For high energy, we observed spectral broadening owing to nonlinear propagation predominantly causing 


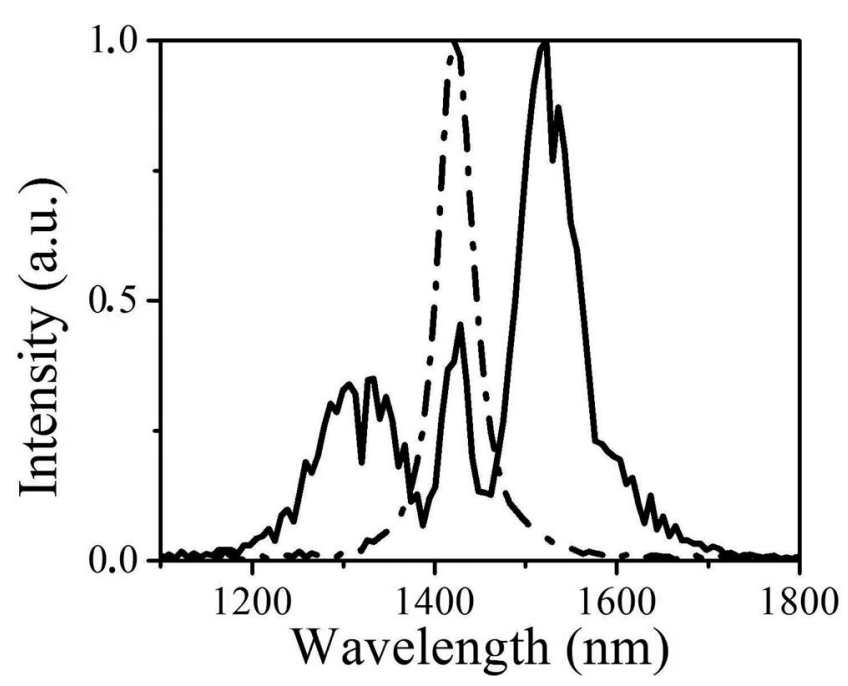

Fig. 1. OPA spectra after propagation into the argon-filled hollow-core fiber as a function of output energy. Dasheddotted curve, $30 \mu \mathrm{J}$; solid curve, broadened spectrum with $400 \mu \mathrm{J}$ corresponding to the $13.1 \mathrm{fs}$ pulse of Fig. 3(b).

SPM. Assuming Fourier transform-limited pulse duration for the broadened spectra, pulse duration at the FWHM of the intensity profile is $12.2 \mathrm{fs}$. At $1425 \mathrm{~nm}$, this is equivalent, in terms of the number of cycles, to $7 \mathrm{fs}$ pulse duration at $800 \mathrm{~nm}$.

To first approximation, the phase accumulated by SPM is linear and positive in the time domain corresponding to a positive group-delay dispersion (GDD) in the spectral domain $[11,12]$. Prism-based negative delay lines introduce significant higher-order dispersion [mainly third-order dispersion (TOD)] being thus unsuitable for few-optical-cycle pulse compression. Consequently, dispersive mirrors were designed to introduce negative GDD and small, negative TOD to compensate for the substantially quadratic chirp carried by the pulses as well as for the positive TOD of glasses eventually used in the setup. We designed and manufactured a pair of dispersive mirrors exhibiting high reflectance $(R>99.5 \%)$ and controlled dispersion $\left(\mathrm{GDD}=-150 \mathrm{fs}^{2}\right.$ and TOD $=-15 \mathrm{fs}^{3}$ at $1425 \mathrm{~nm}$ ) in the wavelength range of $1000 \mathrm{~nm}$ to $1700 \mathrm{~nm}$. Each mirror consists of 73 layers. $\mathrm{SiO}_{2}$ and $\mathrm{Nb}_{2} \mathrm{O}_{5}$ were used as coating materials. The large absolute value of the GDD is a consequence of the increased multilayer thickness (as compared with similar mirrors for the $800 \mathrm{~nm}$ range). Decreasing the number of layers and therewith the overall thickness would result in smaller absolute GDD values at the expense of reduced bandwidth and/or lower reflectance. These design constraints can be overcome by employing materials with a higher index ratio, but the deposition of these materials does not fulfill the necessary thickness accuracy standards yet (to our knowledge). Large GDD (absolute) values per bounce are, however, not a drawback, since they result in a very compact, high-throughput compressor. Fine tuning of the GDD, if necessary, can be achieved with thin positive dispersion glass (e.g., SF10) wedges.

Temporal characterization of the laser pulses was performed using a home-build second-harmonic- gen- eration frequency-resolved optical gating (SHGFROG) [19]. Since phase matching is extremely critical for few-optical-cycle laser pulse measurements, SHG was generated by recombining both FROG optical arms focused using a $50 \mathrm{~cm}$ focal length convex silver mirror inside a $10 \mu \mathrm{m}$ thickness Type I $\beta$-barium borate $(\mathrm{BBO})$ crystal $\left(\theta \approx 21^{\circ}\right)$.

Figure 2 shows, on a linear scale, an experimental (a) and a retrieved (b) SHG-FROG spectrogram for the shortest laser pulse duration we obtained. Dispersion compensation has been achieved by adding one round trip on the chirped mirrors with $\sim 1.5 \mathrm{~mm}$ of SF10. In Fig. 3(a), we present the intensity autocorrelation associated with the experimental SHGFROG traces. The black curve corresponds to the shortest pulse duration, whose FWHM of the autocorrelation is $18.4 \mathrm{fs}$, corresponding to pulse duration of $13.0 \mathrm{fs}$, assuming Gaussian intensity profile. In Fig. 3(b), we are showing the retrieved temporal profile of the laser pulses obtained using the SHG-FROG reconstruction. The fact that the obtained FWHM duration of $13.1 \mathrm{fs}$ is only 1.07 times the Fourier limit (12.2 fs assuming flat spectral phase) proves the good compressibility of the SPM broadened spectra at $1425 \mathrm{~nm}$. The intensity profile is asymmetric with a flat temporal phase covering the main pulse, shown as dashed-dotted curve in Fig. 3(b). Pedestals might origin from uncompensated high-order phase. At $1425 \mathrm{~nm}, 13.1 \mathrm{fs}$ is less than three optical cycles, equivalent to about $7.5 \mathrm{fs}$ pulse duration at $800 \mathrm{~nm}$. Moreover, we have calculated that $70 \%$ of the energy is concentrated in the central part of the laser pulse temporal intensity profile. We have determined the direction of the time axis by overcompensating the dispersion with one round trip on the chirped mirrors and added positive dispersion with SF10 windows.

For comparison with the shortest pulse, a cleaner temporal pulse shape can be obtained by reducing

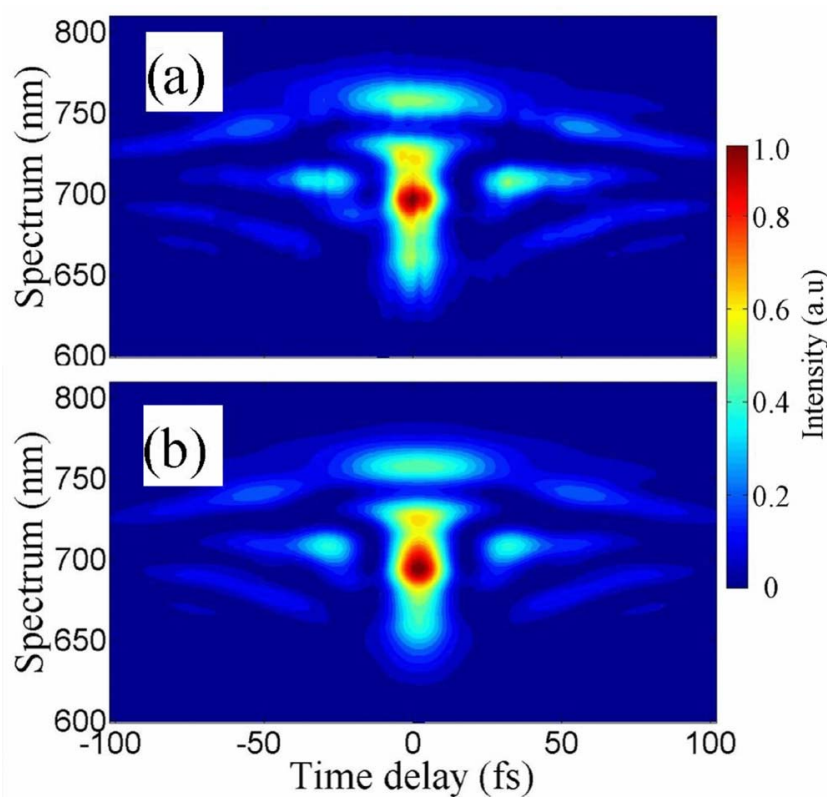

Fig. 2. (Color online) (a) Experimentally measured and (b) retrieved SHG-FROG traces for the compressed $13.1 \mathrm{fs}$ laser pulse of Fig. 3(b). 


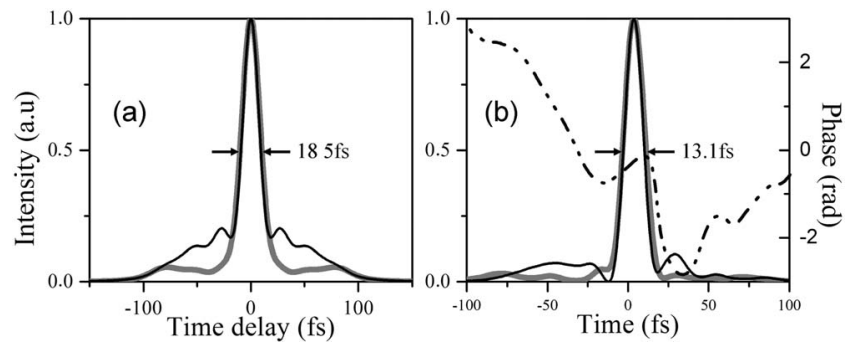

Fig. 3. (a) SHG intensity autocorrelation and (b) retrieved intensity profile and temporal phase obtained from the SHG-FROG reconstruction. The gray curves correspond to the temporally clean $14.5 \mathrm{fs}$ pulse, whereas the black curves show results for the shortest FWHM duration of $13.1 \mathrm{fs}$.

propagation nonlinearity at reduced argon pressure of $1.4 \mathrm{~atm}$ (gray curves in Fig. 3). The retrieved FWHM of intensity is $14.5 \mathrm{fs}$, whereby the main pulse carries more than $86 \%$ of the total energy. This clean pulse was used to experimentally prove the chirped mirror design by measuring an SHG-FROG trace with removed chirped mirrors in the setup. In this case the retrieved spectral phase for the temporally broadened pulse exhibits mainly quadratic behavior (data not shown). From this the second-order dispersion could be determined to be about $-330 \mathrm{fs}^{2}$, which is in very good agreement with calculated value of $-300 \mathrm{fs}^{2}$ for one round trip.

In this Letter, we have shown that hollow-core fiber pulse compression techniques used at $800 \mathrm{~nm}$ can be transferred in the IR. We have generated and characterized $400 \mu \mathrm{J}, 13.1 \mathrm{fs}$ laser pulses at $1425 \mathrm{~nm}$. This is equivalent in terms of number of cycles to $7.5 \mathrm{fs}$ pulse duration at $800 \mathrm{~nm}$. By using a more powerful OPA input [15] and a differentially pumped fiber [12], it should be possible to scale up the output energy. The present pulse duration is limited by the spectral broadening in the fiber owing to the relatively long pulse duration of the OPA, not by the bandwidth of the chirped mirrors. However, the compression of ultrabroadband spectra requires precise dispersion control of TOD by accordingly designed chirped mirrors. At the moment, our fewoptical-cycle IR laser pulses are not CEP stabilized. It will require using a CEP-stabilized Ti:Sa amplifier and using an OPA based on signal seed generated by white light continuum. As clearly explained by Corkum and Krausz [9], generation of CEP-stabilized few-optical-cycle laser pulses in the IR will be highly beneficial for the generation of shorter attosecond laser pulses, to perform high temporal resolution timeresolved molecular orbital tomography combined with high spatial resolution, and for studying recollision physics using electrons with higher energy than what can be provided using Ti:Sa laser technology.

We are very grateful for the help and the time spent on the laser system by François Poitras. The authors acknowledge the support of the Canada
Foundation for Innovation, the Canadian Institute for Photonic Innovations, the Natural Sciences and Engineering Research Council of Canada, and the Fonds Québécois de la Recherche sur la Nature et les Technologies.

\section{References}

1. M. Hentschel, R. Kienberger, Ch. Spielmann, G. A. Reider, N. Milosevic, T. Brabec, P. B. Corkum, U. Heinzmann, M. Drescher, and F. Krausz, Nature 414, 509 (2001).

2. R. Kienberger, E. Goulielmakis, M. Uiberacker, A. Baltuska, V. Yakovlev, F. Bammer, A. Scrinzi, Th. Westerwalbesloh, U. Kleineberg, U. Heinzmann, M. Drescher, and F. Krausz, Nature 427, 817 (2004).

3. I. J. Sola, E. Mével, L. Elouga, E. Constant, V. Strelkov, L. Poletto, P. Villoresi, E. Benedetti, J.-P. Caumes, S. Stagira, C. Vozzi, G. Sansone, and M. Nisoli, Nat. Phys. 2, 319 (2006).

4. G. Sansone, E. Benedetti, F. Calegari, C. Vozzi, L. Avaldi, R. Flammini, L. Poletto, P. Villoresi, C. Altucci, R. Vellota, S. Stagira, S. De Silvestri, and M. Nisoli, Science 314, 443 (2006).

5. F. Légaré, K. F. Lee, I. V. Litvinyuk, P. W. Dooley, A. D. Bandrauk, D. M. Villeneuve, and P. B. Corkum, Phys. Rev. A 72, 052717 (2005).

6. A. Hishikawa, A. Matsuda, M. Fushitani, and E. J. Takahashi, Phys. Rev. Lett. 99, 258302 (2007).

7. E. Goulielmakis, M. Schultze, M. Hofstetter, V. S. Yakovlev, J. Gagnon, M. Uiberacker, A. L. Aquila, E. M. Gullikson, D. T. Attwood, R. Kienberger, F. Krausz, and U. Kleineberg, Science 320, 1614 (2008).

8. P. B. Corkum, Phys. Rev. Lett. 71, 1994 (1993).

9. P. B. Corkum and F. Krausz, Nat. Phys. 3, 381 (2007).

10. J. Itatani, J. Lévesque, D. Zeidler, H. Niikura, H. Pépin, J. C. Kieffer, P. B. Corkum, and D. M. Villeneuve, Nature 432, 867 (2004).

11. S. Sartania, Z. Cheng, M. Lenzner, G. Tempea, Ch. Spielmann, F. Krausz, and K. Ferencz, Opt. Lett. 22, 1562 (1997).

12. A. Suda, M. Hatayama, K. Nagasaka, and K. Midorikawa, Appl. Phys. Lett. 86, 111116 (2005).

13. C. Chudoba, J. G. Fujimoto, E. P. Ippen, H. A. Haus, U. Morgner, F. X. Kärtner, V. Scheuer, G. Angelow, and T. Tschudi, Opt. Lett. 26, 292 (2001).

14. F. Légaré, M. Naji, P. Lassonde, D. Comtois, V. Crozatier, T. Oksenhendler, H. Anis, and J. Kieffer, Opt. Lett. 33, 2824 (2008).

15. E. J. Takahashi, T. Kanai, Y. Nabekawa, and K. Midorikawa, Appl. Phys. Lett. 93, 041111 (2008).

16. X. Gu, G. Marcus, Y. Deng, T. Metzger, C. Teisset, N. Ishii, T. Fuji, A. Baltuska, R. Butkus, V. Pervak, H. Ishizuki, T. Taira, T. Kobayashi, R. Kienberger, and F. Krausz, Opt. Express 17, 62 (2009).

17. C. P. Hauri, R. B. Lopez-Martens, C. I. Blaga, K. D. Schultz, J. Cryan, R. Chirla, P. Colosimo, G. Doumy, A. M. March, C. Roedig, E. Sistrunk, J. Tate, J. Wheeler, L. F. DiMauro, and E. P. Power, Opt. Lett. 32, 868 (2007).

18. C. Vozzi, F. Calegari, E. Benedetti, S. Gasilov, G. Sansone, G. Cerullo, M. Nisoli, S. De Silvestri, and S. Stagira, Opt. Lett. 32, 2957 (2007).

19. R. Trebino, K. W. DeLong, D. N. Fittinghoff, J. N. Sweetser, M. A. Krumbügel, B. A. Richman, and D. J. Kane, Rev. Sci. Instrum. 68, 3277 (1997). 